\title{
«Questo, io penso, e per questo sono contro il divorzio». Il dibattito sul divorzio nella rivista femminile «La Chiosa» (1919-1927)
}

\begin{abstract}
All'indomani della Prima Guerra Mondiale, in un momento di forte crisi socio-politica, la classe dirigente italiana si pose il problema di rettificare le numerose irregolarità coniugali derivate dal conflitto. Nel 1920, i socialisti Guido Marangoni e Costantino Lazzari discussero in Parlamento un progetto di legge sul divorzio, suscitando accese reazioni nell'opinione pubblica. Questo articolo, utilizzando la stampa femminile come documento delle posizioni che le donne assunsero nel dibattito, ripercorre le ragioni e le modalità di espressione dell'antidivorzismo femminile. Quello che si intende verificare è come e con quali strategie le donne proposero la famiglia tradizionale come base di una ricostruzione dell'ordine nel dopoguerra. A questo scopo si sofferma sul settimanale genovese «La Chiosa. Commenti settimanali femminili di vita politica e sociale» (1919-1927), fondato dalla giornalista e scrittrice Flavia Steno. La rivista affronta il tema del divorzio promuovendo un sondaggio tra lettori e lettrici, pubblicando in appendice il romanzo Gli orfani dei vivi di Steno, e commentando l'iter della legge con articoli dedicati. Queste tre diverse modalità di elaborazione di un discorso in difesa della famiglia condividono alcuni aspetti, analizzati in modo specifico e contestualizzati nella delicata fase postbellica italiana: la riattualizzazione del nesso famiglia-nazione, la rappresentazione degli effetti collettivi del divorzio, e la visione puerocentrica del nucleo.
\end{abstract}

The e-journal «altrelettere» is hosted at the URL: http://www.altrelettere.uzh.ch, in accordance with the Open Access Policy of the University of Zurich. Please cite this article as follows: V. IACONIS, «Questo, io penso, e per questo sono contro il divorzio». Il dibattito sul divorzio nella rivista femminile «La Chiosa» (1919-1927), in «altrelettere», (2020), pp. 24-49, DOI: 10.5903/al_uzh-46.

(C) This article is licensed under a Creative Commons Attribution 2.5. Switzerland (CC BY-NC-ND 2.5). Please read the license terms on the website: http://creativecommons.org/licenses/by-nc-nd/2.5/ch/deed.en 


\section{Introduzione}

«Si può dire che da quando esiste il Parlamento italiano, esiste anche un progetto di legge per lo scioglimento del matrimonio» (ATTI DEL PARLAMENTO 1920, 1962). Così, il 6 maggio 1920, il deputato socialista Guido Marangoni iniziava la discussione di una proposta di legge sul divorzio elaborata con Costantino Lazzari. Si tratta del decimo progetto in materia sottoposto al Parlamento dall'Unità di Italia e segna la «fine di un'epoca» (FRANCESCHI 2012, 54) perché è l'ultimo discusso prima del ventennio fascista. Dai suoi predecessori Marangoni riprende alcune cause di divorzio: una separazione di due anni, l'impotenza, la condanna alla lunga carcerazione o all'ergastolo, e l'infermità mentale. Due le novità più vistose: l'articolo 15, che permetteva a entrambi i coniugi di divorziare se questi, rientrati dal fronte o dai luoghi della mobilitazione femminile, avessero scoperto l'adulterio del partner; e l'articolo 16, che disciplinava i casi di bigamia a seguito di morte presunta in guerra. Si trattava di misure necessarie perché, osservava Marangoni, la Prima Guerra Mondiale era stata una débâcle per la famiglia:

\footnotetext{
Oggi l'istituto della famiglia, egregi colleghi, è stato assai compromesso dalla guerra. Che terribile legge per lo scioglimento del matrimonio è stata la guerra! Ha fatto sì che questa riforma si imponesse nei fatti senza temere le opposizioni del partito popolare, che minaccia di insorgere contro la nostra proposta. Ed è per questo che noi [socialisti] dopo avere sempre considerato il divorzio come un lusso snobistico delle classi aristocratiche e privilegiate ci siamo decisi a proporlo quando abbiamo visto sanguinare il problema fra le file istesse dei nostri compagni, dei combattenti proletari i quali tornando dal fronte trovarono distrutta la propria famiglia, e invocano ora straziati il nostro aiuto. (Atti del Parlamento 1920, 1962)
}

La Grande Guerra era stata una 'catastrofe sentimentale': i legami affettivi e familiari si erano spezzati e le tragedie private avevano investito la dimensione pubblica (Thébaud 2001, 58-64; Gabrielli-Montesi 2009, 6-9). Lo Stato italiano era intervenuto a tutela della famiglia e a garanzia della moralità femminile con delle misure straordinarie: il matrimonio per procura (1915), la sospensione dell'autorizzazione maritale per alcune categorie femminili (1917), la ricerca di paternità per gli orfani illegittimi dei militari, e prendendosi carico dell'assistenza all'infanzia. Accorgimenti volti a evitare una crisi della famiglia che, invece, sembra consumarsi proprio nel 1920, quando si verificò, sì, un boom dei matrimoni, ma furono anche approvate 1641 domande di separazione, a cui andrebbero aggiunte quelle ufficiose di entità ignota (Palazzi 
1997, 419). In questo quadro, Lazzari e Marangoni proponevano il divorzio per tagliare i rami secchi dei nuclei non più ricomponibili e regolarizzare quelli illegittimi, così da garantire un istituto familiare stabile ancorché dissolubile, perché conforme alla volontà dei coniugi. Le loro preoccupazioni erano condivise e, infatti, il progetto di legge ottenne buoni riscontri da parte del governo e degli Uffici. Però, a causa della chiusura anticipata della sessione parlamentare (aprile 1921), esso non completò il suo iter e cadde nel dimenticatoio, tanto da essere menzionato solo en passant anche dalla più dettagliata storia del divorzio in Italia ora disponibile, Debating divorce in Italy (Seymour 2006, 161).

A prescindere dalla necessità di riformare il matrimonio, la fragile Italia della fase postbellica temeva le conseguenze dell'introduzione del divorzio sulla stabilità della famiglia. Sin dal Risorgimento all'istituto familiare era stato attribuito un intenso valore identitario, che fu recepito anche nell'Italia liberale e poi fascista (BANTI 2011, IX). La centralità del 'mito' della famiglia nella costruzione della nazione è in effetti una delle ragioni, insieme alla questione cattolica, della lunga gestazione della legge sul divorzio in Italia (SEYMOUR 2006, 223-4). L'idea della famiglia come «sinonimo della comunità nazionale nel suo complesso» e «suo nucleo fondativo minimale» (BANTI 2011, 15) è rilevante anche nel dopoguerra, quando si affermarono delle realtà politiche estranee alla tradizione risorgimentale in cui la classe liberale affondava le sue radici: gli stessi Marangoni e Lazzari militavano nel Partito Socialista di Nicola Bombacci che, insieme al Partito Popolare di Luigi Sturzo, era stato premiato nelle elezioni del novembre 1919. Esse si tennero in un clima di esasperato scontento a cui contribuivano diversi fattori, tra cui la crisi economica e la frustrazione per quella che Gabriele D’Annunzio aveva definito una 'vittoria mutilata'. Questo sentimento, oltre a costituire il sostrato ideologico dell'impresa di Fiume, accomunava un nuovo soggetto politico: gli ex combattenti che, tornati in patria, si sentivano trascurati dalle istituzioni e aspiravano a maggiori riconoscimenti politici e sociali. Uno dei bersagli della loro polemica fu il lavoro femminile, visto come una forma di competizione antipatriottica e fortemente osteggiato, fino a diventare simbolo di una 'guerra tra i sessi' (GrAZIOSI 1995, 26-8). Il modo in cui il conflitto interferì e in parte cambiò le dinamiche di genere fu un ulteriore motivo di disorientamento 
identitario. Mentre gli uomini erano al fronte, le italiane parteciparono alla guerra sostituendoli al lavoro, praticando il volontariato e l'assistenzialismo: «il rimescolamento di ruoli e funzioni produsse una maggiore visibilità delle donne nella sfera pubblica e si riverberò nelle relazioni con gli uomini» (BARTOLONI 2016, 9-10). Questo non solo a livello sociale, ma anche normativo: nel luglio 1919 fu emanata la legge Sacchi, che permetteva alle donne l'accesso agli impieghi pubblici e alle professioni e aboliva l'autorizzazione maritale, correggendo in parte la precedente subordinazione economica delle mogli ai mariti.

Di un simile contesto va tenuto conto nel giudicare la ricezione della proposta di legge sul divorzio di Marangoni e Lazzari. Documento prezioso del dibattito al femminile sul divorzio è la stampa delle e per le donne, il cui numero di pubblicazioni politiche e culturali aumentò notevolmente nel dopoguerra (Mondello 1987, 57-62; ButTAFUOCO 1988, 259-60). Utilizzando la pubblicistica femminile come fonte, intendo occuparmi di alcune delle voci che sono intervenute sul tema e, in particolare, di quelle contrarie al divorzio. Nel periodo liberale l'antidivorzismo femminile era un esito dello stretto legame tra la Chiesa e le donne, e dei loro svantaggi politici, economici e sociali, che le inducevano a considerare l'indissolubilità come una forma di tutela. Su questi aspetti, in modo più o meno esplicito, si soffermavano gli interventi delle donne in materia (SEYMOUR 2006, 7). La disamina del dibattito sulla stampa femminile permetterà di vedere come cambiano gli argomenti portati a detrimento del divorzio negli anni Venti, in cui si riscontra una diversa situazione: le asimmetrie tra moglie e marito nel nucleo erano state in parte riviste, le donne avevano un maggiore accesso all'istruzione e al lavoro, praticavano degli stili di vita più emancipati e, loro malgrado, durante la guerra avevano esperito la solitudine, riformulando le loro aspettative familiari. 


\section{Una «Chiosa» al divorzio negli anni Venti}

Il mio caso di studio sarà la rivista genovese «La Chiosa. Commenti settimanali femminili di vita politica e sociale»(1919-1927). La direttrice, Amelia Osta Cottini (1877-1946), nel 1919 aveva alle spalle un ventennio di carriera giornalistica, iniziata nel 1898 a «Il Secolo XIX» sotto la direzione di Luigi Arnaldo Vassallo, che la scoprì e la ribattezzò Flavia Steno. L'anno successivo esordiva come scrittrice, dando alle stampe il primo di circa novanta volumi tra novelle e romanzi.1 Steno è stata definita una femminista di destra «che non partecipa alle lotte emancipazioniste, rimane alla finestra a guardare, ma si riserva invece il diritto di commento quando non di censura» (SANTINI 2001, 107). Antonella PicchiotTi $(2010,62)$ propone invece di parlare di «afemminismo» per indicare i caratteri disomogenei del pensiero di Steno sulla femminilità: il rifiuto del suffragio femminile è ad esempio compensato dalla rivendicazione del diritto delle donne all'istruzione e al lavoro, mentre all'antidivorzismo si accompagna la critica della doppia morale, la promozione della ricerca di paternità e la diffusione delle teorie neomalthusiane.

Cassa di risonanza delle opinioni di Steno fu «La Chiosa», diffusa soprattutto in Liguria e dalla forte connotazione di genere: la redazione era composta principalmente da giornaliste e la readership era femminile. I contenuti rispondevano a «quell'insieme di curiosità, aspettative, bisogni che si presupponeva fossero nutriti dalle lettrici medie» (FRANCHINI 2000, 126), con attenzione all'attualità e ai nuovi ruoli sociali delle donne, sia in Italia che all'estero (cfr. VERDINO 1988, 307-8). La rivista si prefiggeva un duplice scopo: informare e educare alla vita politica le sue lettrici che - almeno così si credeva - avrebbero presto beneficiato del diritto di voto grazie alla proposta di legge in materia di Francesco Nitti (settembre 1919; cfr. BIGARAN 1987, 258-60). Allo scopo informativo-pedagogico, inoltre, se ne accompagnava uno politico: «La Chiosa» si proponeva come polo di attrazione per un elettorato femminile liberale, nel tentativo di prevenire l'adesione delle donne ai partiti di massa, come risulta dalla corrispondenza tra Steno e Mario e Pio Perrone, finanziatori de «La Chiosa», e in particolare dalle lettere del 21 febbraio e del 12 luglio 1920 (FA FP SSNB 107/15).

Oltre a pubblicare articoli dedicati all'iter burocratico della legge, il settimanale ospitò dal 19 febbraio al 15 aprile del 1920 un 'referendum' sul 
divorzio, per saggiare l'opinione di lettrici e lettori sul tema. Pochi mesi dopo, compariva in appendice il romanzo Gli orfani dei vivi di Steno (16 dicembre 1920-13 ottobre 1921), edito in volume da Treves nel 1926. Il testo occupa un ruolo singolare nella produzione della giornalista e, come «La Chiosa», può essere inteso come parte di un progetto di autoaffermazione professionale. Questa, almeno, è l'impressione che si ricava da una lettera inviata dall'autrice a Ugo Ojetti il 23 marzo 1923 per chiedere una recensione del testo sul «Corriere della Sera». Il libro, afferma Steno, va promosso perché è «un buon libro - nel senso morale e educativo» (FONDO OJETTI). I punti di forza sono la rilevanza dei temi («questo libro [...] è una battaglia contro il divorzio e in difesa dei diritti del figlio», ivi) e la sua efficacia letteraria («l'ho scritto col cuore e mi sembra riuscito», ivi). C'è poi un'altra ragione che preme all'autrice:

Il Corriere aveva lodato il mio primo libro - La nuova Eva - pubblicato nel 1901, con un articolo di una colonna e mezza di Federico de Roberto. Dopo, per tanti anni, circostanze di famiglia (sono capo di casa della famiglia mia e di quella dell'unica mia sorella perduta, sette persone cui provvedere, sola!) mi costrinsero a fare della letteratura... commestibile, appendici che tuttavia curavo con dignità, novelle, articoli. Ora, son quasi vecchia: ho fatto questo romanzo con la speranza di riconquistare un po' del tanto tempo perduto dal punto di vista della serietà del nome. Vi chiedo con umiltà e con fiducia: degnatevi di osservarlo e di farlo osservare; e, se Vi pare lo meriti, concedetegli un po' del Vostro altissimo patrocinio! (ivi)

Non è la prima volta che Steno sottolinea le ragioni economiche che sottostavano alla sua scrittura, "commestibile" appunto perché fonte prima dei mezzi di sostentamento (Picchiotti 2002, 222). Con Gli orfani dei vivi, come il contenuto della lettera evidenzia, l'autrice tenta di rilanciare un'immagine di sé come scrittrice impegnata già a suo tempo proposta con il romanzo La nuova Eva.2 È quindi riduttivo affermare che, attraverso il testo, «l'autrice trasmette la propria esperienza di figlia di coppia separata e che molto ha sofferto di questa condizione [...] con una forte connotazione autobiografica» (Santini 2001, 124-5). Questo giudizio, in linea con un generale riduzionismo biografico della scrittura delle donne, appiattisce sul privato un testo che, invece, non solo è una prova dell'intelligente gestione di Steno della propria immagine di scrittrice e giornalista, ma, soprattutto, ha palesi intenti politici. Sensibilizzare le donne, future elettrici, verso i mali del divorzio è lo scopo ultimo della campagna antidivorzista promossa da Steno attraverso un doppio canale, pubblicistico e narrativo, di cui osserveremo le diverse declinazioni. 
«La questione del divorzio essendo di capitale importanza, La Chiosa apre le sue colonne a tutti quanti vi si appassionano per la più larga discussione possibile sull'argomento»(STENO 1920, 1). Così «La Chiosa» inaugurava il referendum sul divorzio, dimostrando di aver fatto propria sin dai primi mesi di attività la formula del sondaggio, che prevedeva il coinvolgimento del pubblico nell'elaborazione del prodotto-giornale con interventi sull'attualità (DITTRICH-JOHANSEN 1995, 823). Il referendum raccolse 22 contributi inviati da lettori e lettrici e dalle redattrici del settimanale, tra cui la triestina Willy Dias (Fortunata Morpurgo; 1872-1956), la bergamasca Donna Paola (Paola Baronchelli Grosson; 1866-1954) e la cosentina Ester Bonomi. 10 sono i pareri contrari al divorzio e 9 quelli favorevoli, mentre Donna Paola, il giornalista Ernesto Salvatore Arbocò e il medico Antonio Parlati si astengono dal giudizio, proponendo piuttosto una lettura dell'instabilità coniugale come segno di un malessere sociale. Donna Paola, “divorzista pentita”, perché prima del conflitto aveva promosso questa legge, rileva ad esempio come

\footnotetext{
la crisi che travaglia il matrimonio [...] non è tanto dell'istituzione quanto dell'individuo. [...] L'unica cosa consistente, contro la quale si possano avventare gli strali della critica e l'amarezza dei rimproveri è la corruzione dell'individuo, è il suo egoismo, la sua furia di sfruttamento, la sua avidità al piacere, per cui né doveri sanciti da leggi, né doveri rampollati dall'imo della coscienza, hanno nulla a che vedere, anzi sono derisi e vilipesi... (DONNA PAOLA 1920,1$)$
}

Gli interventi evidenziano due approcci diversi al tema: chi si esprime a favore del divorzio lo fa guardando alle singole coppie: «dato e non concesso che $\mathrm{i}$ casi in cui il divorzio appare una necessità impellente siano rari, basterebbero tali casi rari, anzi, vorrei dire, basterebbe un caso solo per reclamarlo» (Ferraris 1920a, 1). I detrattori, invece, si soffermano sugli effetti collettivi della legge, esprimendo il rifiuto di una svolta individualista della società paventata anche in età liberale (Montaldo 2000, 33-4). Il nesso patriafamiglia si dimostra ancora attuale negli anni Venti. Sulla sua falsariga si delinea il corretto rapporto tra Stato e cittadini, che dovrebbero rinunciare a una parte della loro libertà privata per il bene comunitario. Inoltre, Steno costruisce le sue obiezioni al divorzio partendo da una nozione puerocentrica 
della famiglia, che crede legittimata dalle istituzioni: secondo la giornalista il matrimonio civile sarebbe infatti un «contratto a beneficio di terzi: i figli» (Steno 1920, 1). La cornice giuridica in cui il codice Pisanelli (1865) aveva iscritto il rapporto coniugale è considerata funzionale a gestire le conflittualità nell'ottica dell'interesse delle generazioni più giovani $\mathrm{e}$, per estensione, del bene comune. Il divorzio turberebbe l'equilibrio: «la famiglia ha, per presupposto, il figlio: ora, il divorzio tende a favorire i coniugi nei loro pretesi diritti alla felicità, ma non mai il figlio» (Steno 1920, 1).

L'etica del sacrificio rivendicata da Steno e prescritta a entrambi i genitori deriva dall'adesione alle teorie neomalthusiane, e quindi da un'idea della genitorialità come scelta consapevole: «Procreare non è un obbligo; ma consacrare tutte le forze del nostro essere e tutte le energie del nostro spirito alla felicità della piccola vita chiamata dal nostro istinto, dai nostri sensi, dal sentimento nostro, sì» (ivi). È Ester Bonomi a sottolineare la contraddizione in cui cade Steno, invitata a non condannare a priori la cessione di una maggiore autonomia ai singoli, perché anche il controllo delle nascite, come il divorzio, nasce da un solco individualista:

La nostra valorosa direttrice, che parla in nome della religione, afferma infine che procreare non è un obbligo. Ma tanto la religione che la natura, quando non esistano impedimenti patologici che mettano in serio pericolo la vita della madre, non ne vogliono sapere di maltusianismo [sic.]! Dunque? In nome della morale e della civiltà ben venga la riforma. (Bonomi 1920, 1)

Ai genitori, che pianificano le nascite in base alla loro disponibilità ad accogliere la prole, spetta anche giudicare quale sia il migliore ambiente in cui far crescere i figli. Inoltre, secondo la pediatra, l'interesse di questi ultimi è già compromesso dalle possibili relazioni illegittime dei genitori separati: «il danno economico e giuridico che ne hanno le povere creature nate da tali unioni non è certamente inferiore a quello che subiscono i nati dei divorziati» (ivi).

L’idea che i doveri dei genitori verso i figli siano inderogabili è condivisa da tutti i partecipanti, ma il pomo della discordia riguarda come garantire il benessere della prole. Il dibattito si sofferma quindi sulle famiglie allargate, quelle cioè date dalle seconde nozze. Diversi contributi pro-divorzio equiparano queste nuove famiglie a quelle costruite da vedovi e ne ribadiscono la superiorità rispetto alle unioni illegittime: il secondo matrimonio «dà al patrigno o alla matrigna almeno una figura giuridica e delle giuridiche 
responsabilità»(FERRARIS 1920a, 1), altrimenti negate. Gli antidivorzisti, al contrario, accentuano il diritto della prole a vivere nella famiglia biologica e il dovere dei genitori a risolvere i conflitti mantenendo integra l'unione o, tutt'al più, separandosi:

Il divorzio non vi aggiunge [alla separazione] che l'autorizzazione per entrambi i coniugi a contrarre un nuovo matrimonio a tutto danno del figlio o dei figli che verrebbero ad avere un padrigno o una matrigna, nonché dei fratellastri non per colpa della morte ma in grazia del preteso diritto dei propri legittimi genitori a provvedere alla propria felicità. (STENO 1920,1)

Alle parole di Steno fanno eco quelle di Willy Dias, una "divorzista con riserva”, perché ritiene che «la famiglia, la vera famiglia non è rappresentata da un uomo o una donna che vivono assieme senza aver procreato. Quelli sono due amanti o due amici - e possono divorziare o non divorziare secondo il loro gradimento. La famiglia è già una collettività, dove si sagrifica chi ha maggiore la possibilità e il dovere del sagrificio - e c'è una sola vera - la prima che si è fondata - le altre non possono rimpiazzarla ma distruggerla» (Dias 1920, 1). Le nuove unioni, infatti, genererebbero un'incertezza identitaria, perché i figli sarebbero «o relegati in fino alla giovinezza in collegio o in un casa che è la loro ma non è più la loro, dovendo tollerare la presenza continua d'un padre o d'una madre posticcia» (ivi). In più, vedendosi trascurati dai genitori, i figli perderebbero «per sempre ogni idea di santità della famiglia, di rispetto verso coloro che non hanno saputo fare tutto il loro dovere» (ivi, enfasi nell'originale).

Il nucleo familiare è visto come specchio e modello della comunità nazionale e, per questo, è il bene collettivo che deve prevalere e dirigere i comportamenti dei singoli. In questo quadro lo scopo della legge non è di assecondare la libertà individuale, ma di preservare la collettività: per questo il divorzio viene rappresentato come un «elemento disgregatore della società» (STENO 1920, 1). Emanando una simile legge, le autorità dissolverebbero la catena di solidarietà che lega le generazioni e, soprattutto, avallerebbero una condotta nociva alle istituzioni stesse. D’altro canto, afferma Steno:

esiste la diserzione, scusata da taluni con gli stessi argomenti di egoismo personale che militano in favore del divorzio: tuttavia, nessuno si sognerebbe di invocare una legge che autorizzasse gli individui a provvedere alla propria sicurezza personale quando, per la sicurezza collettiva, la Patria chiama i concittadini a difenderla mettendo in giuoco la vita. (ivi) 
Il parallelismo tra il divorzio e il reato della diserzione, particolarmente odiato a ridosso della guerra, è illuminante del rinnovato uso del discorso sulla famiglia in chiave nazionale. I coniugi sono invitati a sopportare gli obblighi dell'indissolubilità per difendere la famiglia, intesa come la base di un ritorno all'ordine:

\begin{abstract}
Il pericolo sta appunto in questo: nel sancire il principio della dissolubilità del vincolo che trova soltanto nel suo carattere di stabilità inviolabile la garanzia della propria autorità. Proprio oggi, mentre si moltiplicano gli attentati all'ordinamento sociale, sancire con una legge la possibilità di sciogliere il vincolo che rappresenta l'integrità della famiglia - solo rifugio rimasto intatto nel cataclisma universale - sarebbe, oltre che grave colpa, irreparabile errore. Ogni giorno la saggezza e l'esperienza dimostrano la necessità di far macchina indietro e da dove cominceremo, a far macchina indietro, se non avremo, per punto d'appoggio, la famiglia $[\ldots]$ ? (ivi)
\end{abstract}

Nelle colonne dell'antisocialista «La Chiosa», la proposta di legge Marangoni-Lazzari appare come un cavallo di Troia del Partito Socialista, pronto a distruggere la cellula prima della nazione per portare a compimento il suo progetto rivoluzionario. Se già in precedenza l'interesse dei socialisti per il divorzio aveva suscitato preoccupazione (Seymour 2006, 113-34), questa non poteva che approfondirsi nel biennio rosso, quando «le speranze millenaristiche, accese e agitate dai socialisti, esplosero con violenza [...] [e] le paure [apocalittiche] credettero di essere sull'orlo di una rivoluzione» (Carocci 2004, 81). Anche da altri interventi al referendum si colgono timori in questo senso, oltre che un generale biasimo della situazione politica. Il direttore del «Domani d'Italia», Umberto Ferraris, osservava:

\footnotetext{
La proposta di legge per il divorzio è portata alla Camera dai rappresentanti del P.U.S., ciò che mi ha fatto ricordare che il divorzio è anche e soprattutto, nel momento in cui viviamo, una questione politica, nella quale da una parte stanno coloro che incuranti della Nazione e soltanto occupati dagli egoismi individuali trovano intanto che il divorzio, minando la famiglia, sarebbe un mezzo come un altro per disorganizzare vieppiù la Nazione, che nell'indivisibilità della famiglia ha uno dei suoi maggiori sostegni. (Ferraris 1920b, 1)
}

Anche il tenente Aurelio Storaci avvertiva il pubblico: «Se veramente noi italiani ancora detentori dei luminosi raggi de la più grande civiltà, non vogliamo cadere nel baratro de la dissolutezza sociale, io credo sia giunta l'ora del pollice verso a tutte queste riforme che portano allo sfacelo» (Storaci 1920, 4). 
Simili contributi riattualizzano una retorica già presente nel secondo Ottocento, quando «il ricorso all'immagine rassicurante della famiglia come contenitore disciplinante o come archetipo di concordia [era] spesso collegato allo spettro della distruzione della proprietà privata, al profilarsi di dottrine miranti a sconvolgere dalle fondamenta il concetto stesso di Stato» (PORCIANI 2006, 21). Nel sondaggio, per sottolineare questa funzione della famiglia, si evidenzia la sua funzione formativa dei futuri cittadini. I valori nazionali venivano insegnati attraverso pratiche educative attive, delegate principalmente alla madre (SANSON 2013, 48-9, D'AMELIA 2005, passim), ma anche in modo indiretto: dai genitori, la prole mutuava i modelli relazionali che avrebbe applicato nella vita adulta. Sul punto si sofferma Lidia Serando:

Chi non vuol rompere la continuità ideale che lega l'oggi all'ieri sul quale si fonda [la nazione], ha interesse a mantenere la famiglia in tutta la sua forza e la sua integrità, ha istintivamente timore del divorzio: mentre si comprende benissimo che questo sia voluto senza tema dai partiti disgregatori, che non vedono alcun bene nella società odierna e pei quali sarebbe la benvenuta quella qualunque esperienza che venisse a mutarla. (Serando 1920, 4)

L'intervento di Serando è il più articolato contributo al referendum perché indaga sia i singoli nuclei coniugali sia gli effetti collettivi del divorzio. L'insegnante genovese osservava come «tutto considerato, preso nella singolarità dei casi, il divorzio appare legittimo e buono, una vera provvidenza» (ivi). Gli effetti positivi del divorzio nelle coppie erano però compensati da quelli, nefasti, sul piano sociale. Il problema risiedeva nell'impossibilità di impedire un eccesso d'individualismo nella fruizione della legge, e, quindi, una degenerazione della società. Il giudizio finale di Serando, sebbene circoscritto al preciso momento storico, è netto:

\footnotetext{
A me basta notare che probabilmente il divorzio non è un bene per la società, almeno per la società come oggi è costituita e come potrebbe migliorarsi domani. [...] La legge civile [...] ha il dovere, se vuol tutelare la civiltà attuale, di rinunciare al divorzio, anche se abbia potuto balenare la possibilità di risolvere immediatamente per suo mezzo una quantità di situazioni imbarazzanti e dolorose. (ivi)
}

Proiettati sul piano collettivo, i danni provocati dal divorzio superano i benefici. Tale prospettiva, insieme alla visione puerocentrica della famiglia, sposta in secondo piano un'analisi della connotazione di genere dei rapporti di 
forza del nucleo, all’interno del quale la posizione subordinata della moglie è di fatto naturalizzata. Ciò avviene, con alcune sfumature, in tutti i 22 contributi al sondaggio, che quindi non mette a tema il modo in cui il divorzio avrebbe influenzato la vita femminile. Questa lacuna non mi sembra derivare dall'impostazione della testata, che era anzi molto attenta alla lettura degli effetti di genere dell'attualità politica e sociale, e guardava con occhi tutt'altro che conservatori alla femminilità. Raramente, infatti, esaltava la maternità o la domesticità, e spesso invece proponeva dei modelli innovativi, dando visibilità alle varie attività femminili nella sfera pubblica, polemizzando con la stigmatizzazione del lavoro delle donne, e valorizzando la loro autorità intellettuale. Invece, l'andamento del sondaggio $\mathrm{mi}$ pare riflettere la frammentaria riformulazione in termini di genere della questione del divorzio da parte del femminismo italiano (GAZZETTA 2018, 210). Il tema pagava le spese dell'adesione di molte militanti al nazionalismo e della sistematica subordinazione delle istanze femminili ai 'destini nazionali', avvenuta anche in virtù dell'elaborazione del mito della guerra come motore dell'emancipazione delle donne (Rossini 2016, 121-4). Una conferma in questo senso proviene da una fine commentatrice dell'epoca, Laura Casartelli Cabrini (1883-1932), responsabile della Rassegna del movimento femminile italiano dell'«Almanacco della Donna Italiana» (Bemporad, 1920-1943):

Basterebbe ricordare i vari atteggiamenti delle associazioni [femminili] nella questione del divorzio per persuadersi che quelle associazioni sempre perplesse, sempre "amletiche" nello sdoppiamento di una volontà fiacca e incerta, sono destinate a non contare più niente. (CASARTElli CABRINi 1921, 241)

Il dopoguerra è un momento di crisi anche per il primo femminismo, svuotato dei suoi contenuti, sempre più frammentato, e inadatto a leggere $\mathrm{i}$ cambiamenti in atto perché privo di un effettivo ricambio generazionale (cfr. Gazzetta 2018, 203-5). Questi fattori rendevano inefficaci le miriadi di attività in cui i vari gruppi erano coinvolti, attentamente osservate anche da «La Chiosa». In particolare, in cinque articoli pubblicati tra il luglio e il novembre 1920 (Anonimo 1920a; 1920b; 1920c; 1920d; Ferrazzi 1920), la rivista supportava la richiesta dei gruppi democratici di posporre la discussione della legge sul divorzio alla concessione del voto alle donne. La richiesta, dice un'anonima in luglio, rispondeva al «più elementare criterio di giustizia» 
(Anonimo 1920b, 1). Questa 'coda' al sondaggio dimostra che la redazione de «La Chiosa» fosse consapevole della rilevanza del divorzio nella vita femminile, ma che il dibattito pubblico sulla questione, almeno nel caso in esame, si nutrisse di temi e argomenti 'neutri' dal punto di vista di genere, forse più emotivamente efficaci: la paura di accelerare un sovvertimento dell'ordine che sembrava essere già in atto, e il riconoscimento all'infanzia di diritti specifici, in linea con l'ottocentesca “scoperta” del mondo infantile (Montesi 2007, 34).

«Questo, io penso, e per questo sono contro il divorzio» (STENO 1920, 1): così Flavia Steno conclude il suo intervento al referendum, affermando non solo le proprie idee, ma anche il proprio diritto di esprimerle in un contesto pubblico. Allo stesso modo, l'adesione alla proposta dei gruppi democrati sottintende la necessità che le donne possano contare, esercitando i diritti di cittadinanza, nella vita della nazione. Difatti, almeno in questa occasione, «La Chiosa» smussava il dichiarato antisuffragismo:

\footnotetext{
Se c'è una ragione che possa giustificare l'estensione dell'esercizio del voto politico alla donna è proprio soltanto questa, di farla intervenire nella discussione e nella elaborazione di tutte le leggi che la riguardano particolarmente o che mirano a modificare l'istituto famigliare, a stabilire intorno all'indirizzo educativo e ai modi di esplicazione del dovere di assistenza e di solidarietà sociale. (Anonimo 1920b, 1)
}

Attraverso la rivendicazione del ruolo politico delle donne, a cui è principalmente rivolta l'opera di sensibilizzazione e informazione di Flavia Steno, il tema del divorzio acquista una dimensione di genere, trascurata nel sondaggio e solo implicita nel romanzo Gli orfani dei vivi.

\section{Dalla parte dei bambini: Gli orfani dei vivi}

Ne Gli orfani dei vivi Steno immagina l'esistenza di una legge sul divorzio intensamente fruita e narra l'infanzia e adolescenza di Doretta Ardenni, turbate dai litigi tra i suoi genitori, Silvia e il medico Carlo. Dopo aver scoperto l'adulterio del marito, Silvia divorzia e si risposa con il proprio avvocato. A sei anni la bambina è collocata presso l'Istituto Susan a Lugano, che accoglie solo figli di divorziati, fornendo loro una sorta di famiglia putativa. Lì Doretta conosce molti bambini che, come lei, sono trascurati dai propri genitori e, tra le 
insegnanti, si lega in modo particolare alla ginevrina Armandina Roslan. Quando Doretta ha quindici anni, il padre, rientrato da un lungo periodo di lavoro nelle colonie africane, la riprende con sé. Il periodo di serenità è brevissimo: Carlo rincontra la sua antica amante e, assecondandone i desideri, decide di far tornare Doretta al collegio per ricoprire il doppio ruolo di studentessa e insegnante. Il dottor Melzi, un amico di famiglia che ha seguito da vicino i trascorsi della ragazza, le consiglia invece di sposarsi con un suo giovane allievo, ottenendo però un netto rifiuto. Il destino di Doretta è segnato: la ragazza ha perso fiducia nei legami familiari e si vota a un'infelice solitudine:

\footnotetext{
- E quanto al formarmi una famiglia, Melzi me ne ha parlato. Ma gli ho detto di no. Credo che sarà no per sempre. Ci ho pensato già. Ma... voi [mamma e papà] m'avete dato una esperienza troppo triste. Come potrei credere a un uomo dopo aver dovuto perdere la fiducia in mio padre? Mio padre e mia madre non mi hanno amata abbastanza da superare per me il loro egoismo, come potrebbe amarmi un estraneo? (Steno 1946, 244)
}

Doretta rientra a pieno diritto nella schiera di quelle che Maria Serena Sapegno (2018) ha chiamato «figlie del padre»: fin dall'inizio, la bambina immagina la sua futura vita di donna sulla scorta della figura paterna: «Doretta quando sarà grande sposerà un uomo bello come il suo babbino, ecco!» (Steno 1946, 6). Quando i genitori si separano, Doretta può vedere il padre solo una volta a settimana: le visite le procurano uno «stato di orgasmo» e la bambina «non vede che per gli occhi di babbino; non parla che di lui; riferisce a lui, attraverso il giovedì sempre sospirato, ogni più piccolo atto della sua piccola esistenza» (ibid., 50). Questa inclinazione non muta negli anni del collegio, quando la sua speranza è quella di riunirsi con Carlo, con il quale intrattiene uno sporadico rapporto epistolare. Come nei casi indagati da Sapegno (2018), anche qui la madre è estromessa dalla relazione padre-figlia e assume un ruolo negativo. La gelosia di Silvia verso l'affetto di Doretta per il padre si esacerba quando scopre che, dopo la separazione, Carlo ha mantenuto la liaison con la Zari e vi ha coinvolto anche la figlia:

La sua [di Silvia] fantasia esasperata dalla risorta gelosia, le dipinge Carlo felice nella ripresa dell'amore, dimentico di lei, vendicato, soddisfatto. Tutto, ella pensa, egli è riuscito a riavere: una casa, una donna e anche la sua bambina. Ma questo è troppo. La bambina, almeno, non deve averla! Prestargliela così perché egli la stacchi a poco a poco da lei e magari, non pago di affezionarsela unicamente le faccia amare la propria amante, no, no, e no! (Steno 1946, 54) 
Doretta è vittima di un'inadeguata gestione del conflitto coniugale: senza capirne le ragioni, «sente che l'ostacolo tra lei e il babbo è la mamma» (ibid., 29), la quale divorzia sapendo che «è l'unico modo, questo, per infliggergli [a Carlo] il solo dolore al quale egli sia sensibile: il dolore di perdere Doretta» (ibid., 59). Non sono solo le scelte materne, però, a iscrivere il rapporto padrefiglia nel segno della mancanza, ma anche quelle di Carlo, che, come la moglie, antepone i propri sentimenti alla bambina. Ad esempio, dopo il secondo matrimonio di Silvia, invece di prendere Doretta con sé, il dottore decide di partire per l'Africa:

\footnotetext{
Invano Melzi aveva tentato di distoglierlo da questo progetto prospettandogli anche il dovere che gli incombeva di non abbandonare Doretta che ormai non aveva più che lui. Doretta era il dovere, ma, restare era il tormento.

- D'altronde - egli aveva concluso - Doretta è ancora tanto piccola! Fin che rimane in collegio, la mia presenza non le potrà mancare molto. Quando sarà grande, oh, allora! (ibid., 123)
}

La partenza di Carlo e l'incertezza del suo ritorno possono ricordare un allontanamento maschile ben più tragico e vivo nella memoria del pubblico del 1920: quello per il fronte. La scelta di Steno di mettere in scena una figlia del padre può essere motivata anche dal momento storico: nel contesto della Grande Guerra «l'unico lutto infantile pubblicamente celebrato e materialmente risarcito dallo Stato fu la perdita del padre» (Guidi 2016, 225). Alle morti dei padri nei campi di battaglia, Steno accosta quelle, simboliche, causate dal divorzio. Allo stesso modo, gli 'orfani dei vivi' vengono posti in relazione a quelli di guerra, un parallelismo efficace nel momento in cui questa categoria sociale riceveva una particolare attenzione istituzionale (ibid., 224). Steno recupera quindi un discorso emotivamente connotato per mettere a tema il rapporto padre-figlia e descrivere così una crisi del potere. L'autrice de Gli orfani dei vivi rappresenta un amore paterno intenso ma precario: non solo prima della partenza, ma anche dopo il rientro dall'Africa, Carlo si ritrarrà dai doveri paterni. L'uomo stavolta è più consapevole dell'ingiustizia che compie verso la figlia e, nel chiederle di tornare all'Istituto Susan, «s'era aspettato una scenata di disperazione» (Steno 1946, 218). Doretta, invece, non si ribella alla decisione del padre, tanto che, dice Carlo a Melzi, «la sua pronta remissione mi ha anzi stupito e quasi deluso come una dimostrazione di poco attaccamento a 
me» (ibid., 225). Incolpando, quasi, Doretta di un amore filiale insufficiente, il padre cerca di deresponsabilizzarsi di una scelta non intimamente sentita, ma obbligata da terzi:

Ma il sacrificio di Doretta era stato imposto dalla Zari come condizione sine qua non della ripresa [della relazione] che tuttavia era stata lei a sollecitare e Ardenni aveva dovuto obbedire. (ibid., 219)

Se, nel paradigma letterario della relazione padre-figlia, l'autorità paterna viene spesso esercitata per ristabilire un sistema patriarcale ed è proprio «alla nuova figura della "figlia del padre" - di solito primogenita, sempre prediletta - che è affidato il compito di ristabilir[lo], dopo aver assunto su di sé ogni colpa» (Sapegno 2018, 13), qui la situazione è capovolta. Il sacrificio di Doretta non ristabilisce l'ordine, ma lo destabilizza del tutto, perché prescritto da un potere labile e influenzato da un femminile nocivo: «la donna per la quale suo padre l'aveva allontanata e alla quale l'aveva sacrificata [era] la stessa che un giorno aveva spezzato tutta la sua famiglia» (Steno 1946, 231). La separazione dal padre interrompe la formazione emotiva di Doretta, e le impedisce di diventare adulta: la ragazza torna proprio a quell'Istituto Susan, che è centro di formazione - a evidenziare la sua maturazione incompleta -, e luogo per antonomasia dell'esclusione dagli affetti. Restando 'figlia', Doretta porta fino in fondo le scelte dei genitori («Padre e madre comprendono che ogni insistenza sarebbe inutile: il destino che essi hanno foggiato con le loro mani si compie», ibid., 245) e il finale de Gli orfani dei vivi allude a una degenerazione dell'ordine familiare in atto ma - lo vedremo - non inevitabile.

Attraverso il fallimentare rapporto padre-figlia, Steno rappresenta una crisi delle istituzioni, che hanno perso il potere di indirizzare i comportamenti individuali verso il bene collettivo. Non è un caso che l'autorità maschile, fulcro dello status quo, sia diminuita e inficiata da due donne che sovvertono l'ordine familiare: in questo modo il romanzo tematizza, seppure in forma implicita, la dimensione di genere del divorzio, legge destabilizzante anche perché conferisce alle donne un potere inedito. È significativo che l'unica divorziata su cui Steno si sofferma dal punto di vista psicologico sia una donna tradita: questa scelta ci dice molto della paura condivisa che il divorzio turbasse i rodati equilibri di genere (SEYMOUR 2005, 303). Steno aveva spesso criticato la doppia 
morale, con degli accenti che ritornano anche ne Gli orfani dei vivi (cfr. STENO 1946, 11-6). In questo caso, però, l'aspetto sottolineato è la concentrazione di Silvia su se stessa e sul proprio dolore, che le fa trascurare i bisogni di Doretta. Anzi, la donna, dotata di uno strumento per punire il marito, lamenta il fatto che chi le sta intorno le consiglia di «dimenticare, passar sopra, perdonare per non privare Doretta del padre» (ibid., 36). È Melzi a delegittimare le ragioni di Silvia, a ricordarle i suoi doveri di madre e, si sarebbe tentati di aggiungere, quelli di moglie: «insomma, non si distrugge una famiglia e, soprattutto, non si priva del padre una creatura soltanto perché una gonnella è passata nella stanza del medico e si è fermata oltre il necessario» (ibid., 10). Con la figura di Silvia, Steno propone un parallelismo tra devianza dalla norma matrimoniale, autoaffermazione e maternità negativa, secondo uno schema ideologico attivo fin dall'inizio del secolo (BABINI 2015, 166-7): la donna non solo utilizza Doretta per danneggiare il marito, ma la trascura e respinge:

Ecco che la mamma, invece di rispondere all'invito [all'abbraccio] della sua piccola, si ferma in mezzo alla stanza e si copre gli occhi. [...]

- Non posso vederla! Persin nel modo di tendere le braccia somiglia a suo padre! (STENO $1946,7)$

Come nel sondaggio, anche nel romanzo il divorzio è tematizzato considerando la comunità e senza soffermarsi sui singoli casi. Inoltre, lo sguardo che filtra la realtà è quello infantile: sono gli ospiti dell'Istituto Susan a raccontare i trascorsi dei propri genitori, mentre la crisi coniugale degli Ardenni è principalmente narrata dal punto di vista di Doretta. Ciò permette una banalizzazione delle cause di separazione. Essa è funzionale al messaggio antidivorzista, ma sminuisce i gravi disagi materiali e psicologici causati soprattutto alle donne dall'indissolubilità matrimoniale (Palazzi 1997, 94-112). Questi erano stati oggetto di romanzi come Avanti il divorzio (1902) di Anna Franchi e Una donna (1906) di Sibilla Aleramo, che Steno conosceva bene per averli severamente recensiti sul «Secolo XIX» (Picchiotti 2010, 91-2). Simili testi, come altre opere d'autrice pro-divorzio, oltre a narrare dei casi esemplari, esplicitavano gli effetti penalizzanti delle leggi sulle donne (Iaconis 2020, passim). Al punto di vista femminile Steno oppone quello infantile, in base al quale offre una rilettura del diritto di famiglia. Centrale è la scena della morte per incuria del convittore Boby, deplorata dalla direttrice Susan, da Melzi e dal 
dottor Engels. Nell'episodio si esplicitano le tesi antidivorziste dell'autrice, che abbiamo in parte già visto occupandoci del sondaggio: la dimensione religiosa, l'esperienza diretta dei danni ai bambini e, da parte di Engels, questioni «oserei dire giuridiche»:

\footnotetext{
- Sì; io mi sono chiesto tante volte se lo Stato, la legge, l'autorità civile, insomma abbia il diritto di sciogliere ciò che ha una volta legato per dei fini precisi che a un tratto non vengono più presi in considerazione. Mi spiego. Il matrimonio civile non ha ragione di essere che in vista del figlio, della famiglia: è, insomma, un contratto fatto a beneficio di un terzo. Quando questo terzo - il figlio - è venuto, può lo Stato trascurarlo e trascurarne l'opinione nel pronunziare lo scioglimento del contratto che era stato fatto, ripeto, precisamente per tutelarne i diritti nella eventualità della sua venuta? (STENO 1946, 163)
}

La storia di Doretta, invece, testimonia l'incapacità della legge di tenere conto delle necessità e inclinazioni dei bambini, un tema ancora attuale (Pitch 1998, 135-40). La bambina, lasciata all'oscuro dei progetti dei propri genitori, è costretta a separarsi dal padre amatissimo da una «legge, saggia e cieca, inconsapevole dei misteri dei piccoli cuori infantili»(Steno 1946, 40). Steno evidenzia il fatto che la legge trascura i bisogni delle generazioni più giovani, assecondando, invece di correggere, l’individualismo dei genitori. Il fatto che la famiglia sia un coacervo di interessi contrastanti e di diverso rilievo è ribadito in una conversazione tra Doretta e Guido, uno smaliziato ospite del collegio:

- E quand'è che divorziano? [- chiese Doretta]

- Quando non sono più contenti di stare insieme.

- Ma perché non sono più contenti se sono il papà e la mamma?

- O bella! Perché il papà si innamora di un'altra donna e la mamma di un altro uomo.

Doretta inarcò le sopracciglia.

- E se i bambini non vogliono?

- Dio, che sciocchina sei! - osservò Guido Marisi in una risata - cosa vuoi che contino i

bimbi? (ibid., 113)

Come le parole ingenue di Doretta evidenziano, ai genitori viene negata una dimensione sentimentale e personale al di fuori della famiglia: è unicamente dal loro essere 'mamma' e 'papà' che possono trarre una gratificazione emotiva. Se però si rende il matrimonio dissolubile, anche i rapporti verticali acquistano in fragilità, appunto perché si legittimano altre forme e fonti di appagamento. Dell'avvenuta precarizzazione delle figure genitoriali Doretta si rende presto conto e, infatti, si affretta a chiedere rassicurazioni a Melzi sull'amore di Carlo: 
- E adesso anche papà sposerà un'altra donna e vorrà bene agli altri bambini. $\mathrm{E}$ io non avrò più che te, nonno Melzi. [...]

- Ti assicuro, Doretta, che papà tuo non prenderà un'altra moglie, non comprerà degli altri bambini, non darà a Doretta né una matrigna né dei fratellastri. Mi credi? (ibid., 151)

Il verbo 'comprare' è usato nel romanzo in modo ricorrente per intendere il concepimento: rende così l'innocenza del linguaggio infantile, ma soprattutto allude alla reificazione dei bambini e alla loro sostituibilità. La degenerazione sociale indotta dal divorzio è descritta con una rappresentazione a tinte fosche delle famiglie allargate. Gli orfani dei vivi sono tali perché in nessun caso i genitori riescono a includere i figli di primo letto nei nuclei di nuova formazione. Così l'autrice intende dimostrare la fragilità di modelli familiari alternativi a quello tradizionale e la labilità dei legami parentali, che devono pertanto essere tutelati dalle istituzioni. La storia dell'insegnante Armandina è esemplare: «l'una el'altro dei suoi aveva ricostrutto per proprio conto la propria vita dimentichi entrambi di lei, povero avanzo di naufragio abbandonato alla deriva» (ibid., 69). Al disinteresse o debolezza dei genitori biologici si aggiunge poi la crudeltà di quelli acquisiti: anche qui, come nella tradizione favolistica, matrigne e patrigni incarnano il polo negativo della genitorialità (Cordiano 2016, 260-3). Significativa è la storia della piccola Greti, scacciata sia dal patrigno, che non voleva a casa «la figlia dell'altro», sia dalla matrigna «un'americana assai eccentrica che quando aveva saputo della esistenza di Greti aveva commentato: - Très-bien; mais cela regarde sa mère, chéri!» (Steno 1946, 83). «Tra i due egoismi» commenta Steno «Greti cresceva come se i suoi genitori fossero sotterra» (ivi). La scoraggiante carrellata delle esperienze degli ospiti del collegio illustra le possibili conseguenze del divorzio che, assecondando gli adulti nel contesto coniugale e legittimando le seconde nozze, permette loro di abdicare ai doveri genitoriali. Prima ignorata e poi espulsa, la prole non interiorizza un corretto modello familiare, ed è quindi destinata alla devianza o alla solitudine, come Doretta.

Non è però del tutto escluso un ripristino dell'ordine: al rifiuto di Doretta del matrimonio fanno da contraltare le nozze di Armandina, personaggio che diversi segnali del testo (la condizione familiare, il legame con il collegio, la sfiducia nella famiglia), ci invitano a vedere come una versione adulta della sua pupilla. Con il matrimonio l'insegnante supera la condizione di figlia slanciandosi verso «un avvenire diverso assai da quello di rassegnata 
accettazione della sua povera sorte di diseredata buttata in margine della vita» (ibid., 185). La famiglia futura assume un valore risarcitorio («non aveva avuto la famiglia che aveva diritto di pretendere, ma in cambio il destino le dava questa gioia di potersela creare» ibid., 187-8) ma, soprattutto, indica la possibilità che gli errori di padri e madri siano corretti dalle nuove generazioni. Saranno loro a fare "macchina indietro" con un responsabile recupero di quell'indissolubilità familiare prima istituzionalmente sancita e adesso lasciata all'arbitrio del singolo.

\section{Conclusioni}

Questa panoramica della ricezione della proposta di legge MarangoniLazzari su «La Chiosa» si proponeva di restituire un tassello della storia del divorzio in Italia, osservando le ragioni che animarono le antidivorziste nel 1920. La voce cui è stata data più attenzione è quella di Flavia Steno, direttrice della rivista in esame, promotrice del referendum e autrice de Gli orfani dei vivi, cui affida un rilancio della propria attività di scrittrice. Attraverso l'analisi di diverse modalità di trasmissione del messaggio antidivorzista (il dialogo mediato del sondaggio, gli articoli della 'coda' e la finzione narrativa) sono state individuate delle linee comuni: la valorizzazione del nesso famiglia-nazione, messa in atto cercando di immaginare le conseguenze del divorzio sulla collettività; il rilievo dato ai figli, posti al centro del dispositivo famiglia; e la mancata tematizzazione degli aspetti di genere del divorzio. Quest'ultimo aspetto è certamente spiazzante se si pensa al forte sbilanciamento verso la femminilità del settimanale, sia nella composizione della redazione, sia nell'articolazione dei contenuti. Tuttavia, il fatto che in nessuno degli interventi (neanche in quelli pro-divorzio) gli effetti della legge sulle donne vengano discussi, mi lascia pensare che la lacuna sia da imputarsi almeno in parte all'involuzione del primo femminismo che, avendo perso la sua incisività, non riuscì a riformulare il tema a parte subiecti.

Più forza, nella delicata fase postbellica e in pieno biennio rosso, acquisiva invece il discorso nazional-patriottico sulla famiglia intesa come base per la ricostruzione dell'ordine. Tuttavia, dal sondaggio de «La Chiosa» e dal romanzo, emerge un nucleo familiare innervato di relazioni di potere 
asimmetriche sull'asse verticale. Genitori e figli hanno bisogni diversi, spesso opposti, e lottano con armi impari. L’indissolubilità, quindi, viene rappresentata come una forma di tutela dell'infanzia, prima vittima di una deriva individualista della società. Vale la pena però di rilevare che questo assunto si giova anche di prospettive non tradizionali come, per esempio, l'adesione alle teorie neomalthusiane. La rilettura del nucleo dimostra quindi non un'acritica difesa dello status quo, ma la difficile ricerca di un equilibrio tra l’intensificazione affettiva dei rapporti familiari e la loro dimensione pubblica. La posta in gioco è quella di salvaguardare il bene comune attraverso una corretta trasmissione alle nuove generazioni dei valori nazionali. Ł̀ questo aspetto che, più degli altri, le istituzioni dovevano tutelare, a scapito del benessere di quei "pochi” individui che vivevano una situazione coniugale irregolare, su cui intendevano invece intervenire Marangoni e Lazzari. Di qui i riferimenti, nel sondaggio, all'appartenenza partitica dei due firmatari del progetto di legge, considerato come parte di un progetto socialista rivoluzionario.

La paura di un cambiamento imminente che potesse cancellare la tradizione risorgimentale e liberale anima molti dei contributi al sondaggio de «La Chiosa», che si arroccano su una difesa della famiglia vista come custode delle radici anche identitarie della nazione. Con il senno di poi, sappiamo non solo che i pericoli legati all'ascesa del Partito Socialista furono sopravvalutati, ma anche che la vera minaccia risiedeva nella 'sana reazione' del fascismo di Benito Mussolini. Neanche la nostra contemporaneità, però, in cui si assiste a un revival delle destre e dei populismi, è immune da una simile retorica della famiglia, ancora funzionale ad agire da collante nei momenti di crisi sociale e identitaria: «Difenderemo Dio, la Patria e la famiglia» tuona Giorgia Meloni, leader di Fratelli d'Italia, durante la manifestazione di destra "Orgoglio italiano" del 19 ottobre 2019 (Roma) «e fatevene una ragione!» (MELONI 2019). 
Note

1 Per informazioni sulla vita e le attività di Flavia Steno cfr. STOLFI 2007, PicchiotTi 2010 e il numero a lei dedicato de «La Riviera Ligure» (AA.VV. 2010). Su «La Chiosa» si vedano PICCHIOTTI 2002, DE NICOLA 2002 e 2006.

${ }_{2}$ Su La nuova Eva (Sandron, Milano-Palermo-Napoli, 1904) cfr. FrAU 2011, IACONIS 2017, e PINOIA 2019.

\section{Bibliografia}

Fonti primarie

ANONIMO 1920a:

ANonimo, Voto alle donne e divorzio alla Camera dei deputati, in «La Chiosa», II, 21 (8 luglio 1920), p. 1.

ANONIMO 1920b:

ANONImo, Si interroghi la donna (Per la vittoria del divorzio agli uffici), in «La Chiosa», II, 22 (15 luglio 1920), p. 1.

ANONIMO 1920c:

ANONIMO, In tema di voto e di divorzio, in «La Chiosa», II, 32 (5 agosto 1920), p. 3.

ANONIMO 1920d:

ANONImo, I pericoli sociali del divorzio, in «La Chiosa», II, 48 (25 novembre 1920), p. 1.

BONOMI 1920:

Ester BonOMI, La dottoressa Ester Bonomi, in «La Chiosa», II, 11 (11 marzo 1920), p. 1.

DIAS 1920:

Willy DiAS, Willy Dias, in «La Chiosa», II, 12 (18 marzo 1920), p. 1.

DONNA PAOLA 1920:

Donna PaOla, Parla “Donna Paola”, in «La Chiosa», II, 11 (11 marzo 1920), p. 1.

FERRAZZI 1920:

Rosa FERrazzI, Ci imporranno il divorzio?, in «La Chiosa», II, 34 (19 agosto 1920), p. 1.

FERRARIS 1920a:

Mario FERRARIS, Il divorzio, in «La Chiosa», II, 10 (4 marzo 1920), p. 1.

FERRARIS 1920b:

Umberto FERRARIS, Umberto Ferraris Direttore del "Domani d'Italia", in «La Chiosa», II, 12 (18 marzo 1920), p. 1.

SERANDO 1920:

Lidia SERANDO, Le facce del prisma, in «La Chiosa», II, 15 (8 aprile 1920), p. 4.

STENO 1920:

Flavia STENo, Il divorzio, in «La Chiosa», II, 8 (19 febbraio 1920), p. 1. 


\section{STENO 1946:}

Flavia STENO, Gli orfani dei vivi, Milano, Garzanti, 1946.

STORACI 1920:

Aurelio STORACI, ${ }^{* * *}$, in «La Chiosa», II, 15 (8 aprile 1920), p. 4.

Materiali di archivio

FA FP SSNB 107/15

Fondazione Ansaldo, Fondo Perrone, Scatole a Numerazione Blu, 107, fasc. 15.

FONDO OJETTI

Fondo Ojetti, serie 2, cassetta 107, unità archivistica "Steno Flavia, scrittrice".

Atti del Parlamento

ATTI DEL PARLAMENTO 1920:

Atti del Parlamento Italiano - Discussioni della Camera dei Deputati, XXV Legislatura - Sessione 1919-1920 (22/o3/1920 - o8/o5/1920), Roma, Tipografia Camera dei Deputati, 1920, 2 voll., pp. 1962-3.

Studi

AA.VV. 2010:

AA.VV., Flavia Steno. Numero monografico, in «La Riviera Ligure», XXI, 61/62 (2010).

BABINI 2015:

Valeria P. BABINI, Between Public and Private. Sexuality and Maternity in Three 'New Women': Sibilla Aleramo, Maria Montessori and Linda Murri, in Italian Sexualities Uncovered (1789-1914), a c. di Valeria P. Babini, Chiara Beccalossi e Lucy Riall, Basingstoke, Palgrave Macmillan, 2015, pp. 162-81.

BANTI 2011:

Alberto Maria BANTI, Sublime madre nostra: la nazione italiana dal Risorgimento al fascismo, Roma-Bari, Laterza, 2011.

BARTOLONI 2016:

Stefania BARTOLONI, Introduzione, in La Grande Guerra delle italiane. Mobilitazioni, diritti, trasformazioni, a c. di Stefania Bartoloni, Roma, Viella, 2016, pp. 7-20.

BIGARAN 1987:

Mariapia BIGARAN, Il voto alle donne in Italia dal 1912 al fascismo, in «Rivista di storia contemporanea», 16, 1 (1987), pp. 240-65.

BUTTAFUOCO 1988:

Annarita BUTTAFUOCO, Cronache femminili: temi e momenti della stampa emancipazionista in Italia dall'Unità al fascismo, Arezzo, Università degli Studi di Siena, 1988.

CAROCCI 2004:

Giampiero CAROCCI, Destra e sinistra nella storia d'Italia, Roma-Bari, Laterza, 2004. 
Laura CASARTElli CABRINI, Rassegna del movimento femminile italiano, in «Almanacco della donna italiana», Firenze, Bemporad, 1921, pp. 241-70.

CORDIANO 2016:

Alessandra Cordiano, Archetypes and Family Models, in Fables of the Law: Fairy Tales in a Legal Context, a c. di Daniela Carpi e Marett Leiboff, Berlino, De Gruyter, 2016, pp. 259-73.

D'AMELIA 2005:

Marina D’Amelia, La mamma, Bologna, Il Mulino, 2005.

DE NICOLA 2002:

Francesco DE Nicola, Willy Dias e Flavia Steno, scrittrici a Genova, in La fama e il silenzio. Scrittrici dimenticate del primo Novecento, a c. di Francesco De Nicola e Pier Antonio Zannoni, Venezia, Marsilio, 2002, pp. 19-30.

DE NICOLA 2006:

Francesco DE Nicola, Un settimanale femminile di primo Novecento: «La Chiosa», in Giornali delle donne, giornali per le donne, a c. di Francesco De Nicola e Pier Antonio Zannoni, Venezia, Marsilio, 2006, pp. 35-47.

DITTRICH-JOHANSEN 1995:

Helga DitTrich-JohAnsen, La "donna nuova" di Mussolini tra evasione e consumismo, in «Studi storici», 3 (1995), pp. 811-43.

FRANCESCHI 2012:

Fabio FRANCESCHI, I progetti per l'introduzione del divorzio in Italia in epoca postunitaria, in «Stato, Chiese e pluralismo confessionale, 34 (2012), pp. 1-60.

FRANCHINI 2000:

Silvia FrANCHINI, Stampa femminile e stampa di consumo: dalle definizioni ai problemi storiografici, in «Passato e presente», 51 (2000), pp. 123-36.

FRAU 2011:

Ombretta FRAU, La Nuova Eva di Flavia Steno e il romanzo di genere: un bel caso d'apostasia femminile, in Sottoboschi letterari: sei case studies fra Otto e Novecento: Mara Antelling, Emma Boghen Conigliani, Evelyn, Anna Franchi, Jolanda, Flavia Steno, a c. di Ombretta Frau e Cristina Gragnani, Firenze, Firenze University Press, 2011, pp. 143-165.

GABRIELLI-MONTESI 2009:

Patrizia GABRIELli - Barbara MONTESI, Catastrofi sentimentali: eventi. luoghi, soggetti, in «Storia e problemi contemporanei», 52 (2009), pp. 5-19.

GAZZETTA 2018:

Liviana GAZZETTA, Orizzonti nuovi. Storia del primo femminismo in Italia (18651925), Roma, Viella, 2018.

GRAZIOSI 1995:

Mariolina GRAZIOSI, Gender Struggle and the Social Manipulation and Ideological Use of Gender Identity in the Interwar Years, in Mothers of Invention. Women, Italian Fascism, and Culture, a c. di Robin Pickering-Iazzi, Minneapolis, University of Minnesota Press, 1995, pp. 26-51.

GUIDI 2016:

Laura GuIDI, La mobilitazione dellinfanzia, in La Grande Guerra delle italiane. Mobilitazioni, diritti, trasformazioni, a c. di Stefania Bartoloni, Roma, Viella, 2016, pp. 213-28.

IACONIS 2017: 
Valeria IACONIS, Le catene moderne della nuova Eva. La critica della letteratura femminile al codice Pisanelli, in «Chronica Mundi», 12 (2017), pp. 147-78.

IACONIS 2020:

Valeria Iaconis, "Finché legge non vi separi". Il tema del divorzio nella narrativa d'autrice tra Otto e Novecento, Parigi, Garnier, [in stampa, prev. 2020].

MONDELLO 1987:

Elisabetta Mondello, La nuova Italiana. La donna nella stampa e nella cultura del ventennio, Roma, Editori Riuniti, 1987.

MONTALDO 2000:

Silvano MonTALDO, Il divorzio: famiglia e nation building nell'Italia liberale, in «Il Risorgimento», LII, 1 (2000), pp. 5-57.

MONTESI 2007:

Barbara MoNTESI, Questo figlio a chi lo do? Minori, famiglia, istituzioni (1865-1914), Milano, Franco Angeli, 2007.

PALAZZI 1997:

Maura PALAZZI, Donne sole. Storia dell'altra faccia dell'Italia fra società di antico regime ed età contemporanea, Milano, Mondadori, 1997.

PICCHIOTTI 2002:

Antonella PiCCHIOTTI, La nascita di una rivista femminile del primo dopoguerra: La Chiosa di Flavia Steno (1919-1927), in "Giornale di storia contemporanea», 1 (2002), 216-39.

PICCHIOTTI 2010:

Antonella PicChiotTi, Flavia Steno. Una giornalista, una donna (1875-1946), Genova, Fratelli Frilli Editori, 2010.

PINOIA 2019:

Valentina PINOIA, Storie di donne ribelli: il Bildungsroman al femminile in Germania, Inghilterra, Francia e Italia (1900-1914), Roma, Aracne, 2019.

PITCH 1998:

Tamar Piтch, Un diritto per due. La costruzione giuridica di genere, sesso e sessualità, Milano, Il Saggiatore, 1998.

PORCIANI 2006:

Ilaria PoRCIANI, Famiglia e nazione nel lungo Ottocento, in Famiglia e nazione nel lungo Ottocento italiano: modelli, strategie, reti di relazioni, a c. di Ilaria Porciani, Roma, Viella, 2006, pp. 15-53.

RosSINI 2016:

Daniela RossinI, Il Consiglio nazionale delle donne italiane: affinità e contrasti internazionali, in La Grande Guerra delle italiane. Mobilitazioni, diritti, trasformazioni, a c. di Stefania Bartoloni, Roma, Viella, 2016, pp. 112-129.

SANSON 2013:

Helena SANSON, 'La madre educatrice' in the Family and in Society in PostUnification Italy: The Question of Language, in Women and Gender in PostUnification Italy, a c. di Helena Sanson e Katherine Mitchell, Bern, Peter Lang, 2013, pp. 39-64.

SANTINI 2001:

Iasmina SANTINI, Una femminista di destra: Flavia Steno, in Vivere da protagoniste. Donne tra politica, cultura e controllo sociale, a c. di Patrizia Gabrielli, Roma, Carocci, 2001, pp. 107-29. 


\section{SAPEGNO 2018:}

Maria Serena SAPEgno, Figlie del padre. Passione e autorità nella letteratura occidentale, Milano, Feltrinelli, 2018.

SEYMOUR 2005:

Mark SEYMOUR, Keystone of the patriarchal family? Indissoluble marriage, masculinity and divorce in Liberal Italy, in «Journal of Modern Italian Studies», 10, 3 (2005), pp. 297-313.

SEYMOUR 2006:

Mark SEYMour, Debating Divorce in Italy. Marriage and The Making of Modern Italians 1860-1974, New York, Palgrave MacMillian, 2006.

STOLFI 2007:

Valeria Stolfi, La collaborazione giornalistica di Flavia Steno con il «Secolo XIX»e "La Chiosa». Vicende accadute in Svizzera, in Francia e in Italia a partire dalla fine del secolo XIX fino al decorrere del 1927, Milano, Lampi di stampa, 2007.

THÉBAUD 2001:

François THÉBAUD, La Grande Guerra: età della donna o trionfo della differenza sessuale?, in Storia delle donne. Il Novecento, a c. di Georges Duby e Michelle Perrot, Roma-Bari, Laterza, 2001, pp. 25-90.

VERDINO 1988:

Stefano VERDINO, La cultura tra le due guerre, in La letteratura ligure. Il Novecento, vol I, a c. di Giorgio Bertone e Eugenio Buonaccorsi, Genova, Costa \& Nolan, 1988, pp. 270-379.

Siti web

MELONI 2018:

$<$ https://www.giorgiameloni.it/2019/10/19/il-discorso-integrale-di-giorgia-meloni-in-piazzasan-giovanni-a-roma/> sito web ufficiale di Giorgia Meloni (02.06.2020). 